\title{
Parallel Approximate Finite Element Inverses on Symmetric Multiprocessor Systems
}

\author{
Konstantinos M. Giannoutakis and George A. Gravvanis \\ Department of Electrical and Computer Engineering, School of Engineering, \\ Democritus University of Thrace, 12, Vas. Sofias street, GR 67100 Xanthi, Greece \\ \{kgiannou, ggravvan\}@ee.duth.gr
}

\begin{abstract}
A new parallel normalized optimized approximate inverse algorithm for computing explicitly approximate inverses, is introduced for symmetric multiprocessor (SMP) systems. The parallelization of the approximate inverse has been implemented by an antidiagonal motion, in order to overcome the data dependencies. The parallel normalized explicit approximate inverses are used in conjuction with parallel normalized explicit preconditioned conjugate gradient schemes, for the efficient solution of finite element sparse linear systems. The parallel design and implementation issues of the new algorithms are discussed and the parallel performance is presented, using OpenMP. The speedups tend to the upper theoretical bounds for all cases making approximate inverse preconditioning suitable for SMP systems.
\end{abstract}

\section{Introduction}

Sparse matrix computations, which have inherent parallelism, are of central importance in computational science and engineering computations and are the most time-consuming part. Hence research efforts were focused on the production of efficient parallel computational methods and related software suitable for multiprocessor systems, [12/310].

Until recently, direct methods have been effectively used, but the increase of size, even with the use of modern computer systems, has become a barrier to such methods, 2310]. Additionally, the solution of sparse linear systems, because of its applicability to real-life problems, is obtained by iterative methods, which are in competitive demand after the emergence of Krylov subspace methods, 48].

An important achievement over the last decades is the appearance and use of Explicit Preconditioned Methods, 4], for solving sparse linear systems, and the preconditioned form of a linear system $A u=s$ is $M A u=M s$, where $M$ is preconditioner, 24810. The preconditioner $M$ has therefore to satisfy the following conditions: (i) $M A$ should have a "clustered"spectrum, (ii) $M$ can be efficiently computed in parallel and (iii) finally " $M \times$ vector" should be fast to compute in parallel, 248910.

Hence, the derivation of parallel methods was the main objective for which several families of parallel inverses are proposed. The main motive for the derivation of the parallel explicit approximate inverse matrix algorithms is that they 
result in parallel iterative methods in conjunction with parallel preconditioned conjugate gradient - type schemes respectively, for solving finite element linear systems on SMP systems. The important feature of the proposed parallel approximate inverse preconditioning is that the approximate inverse is computed explicitly and in parallel, eliminating the forward-backward substitution, which does not parallelize easily, [10.

For the implementation of the parallel programs, the OpenMP application programming interface has been used. OpenMP has emerged as a shared-memory programming standard and it consists of compiler directives and functions for supporting both data and functional parallelism. The parallel for pragma with static scheduling has been used for the parallelization of loops on both the construction of the approximate inverse and the preconditioned conjugate gradient scheme.

\section{Parallel Explicit Approximate Inverses}

Let us consider the arrow - type linear system, i.e.,

$$
A u=s,
$$

where $A$ is a sparse arrow - type $(n \times n)$ matrix of the following form:

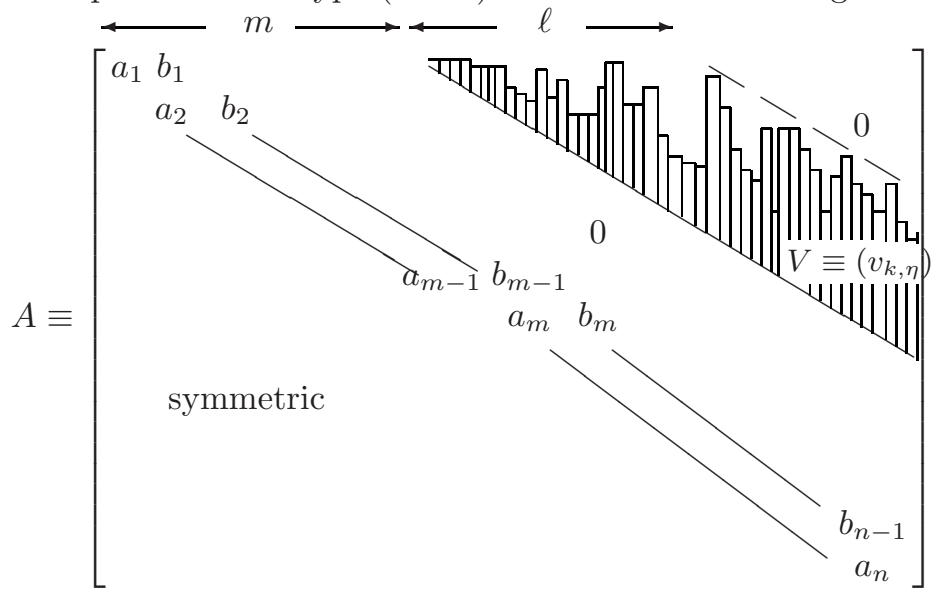

Let us assume the normalized finite element approximate factorization of the coefficient matrix $A$, such that:

$$
A \approx D_{r} T_{r}^{t} T_{r} D_{r}, \quad r \in[1, \ldots, m-1),
$$

where $r$ is the "fill-in" parameter, i.e. the number of outermost off-diagonal entries retained in semi-bandwidth $m, D_{r}$ is a diagonal matrix and $T_{r}$ is a sparse upper (with unit diagonal elements) triangular matrix of the same profile as the coefficient matrix $A, 7$. 
The elements of the $D_{r}, T_{r}$ decomposition factors were computed by the FEANOF algorithm, cf. [12]. The memory requirements of the FEANOF algorithm are $\approx(r+2 \ell+2) n$ words, while the computational work for $m<<n$ is $\approx 1 / 2(r+\ell)(r+\ell+3) n$ mults $+n$ square roots, cf. [7.

Let $M_{r}^{\delta l}=\left(\mu_{i, j}\right), i \in[1, n] j \in[\max (1, i-\delta l+1), \min (n, i+\delta l-1)]$, be the normalized approximate inverse of the coefficient matrix $A$, i.e.

$$
M_{r}^{\delta l}=D_{r}^{-1}\left(T_{r}^{t} T_{r}\right)^{-1} D_{r}^{-1}=D_{r}^{-1} \widehat{M}_{r}^{\delta l} D_{r}^{-1}, \text { with } \widehat{M}_{r}^{\delta l}=\left(T_{r}^{t} T_{r}\right)^{-1} .
$$

The elements of the approximate inverse were determined by retaining a certain number of elements of the inverse, i.e. only $\delta l$ elements in the lower part and $\delta l-1$ elements in the upper part of the inverse (by applying the so-called "position-principle"), next to the main diagonal, by the Normalized Optimized Banded Approximate Inverse Finite Element Matrix -2D algorithmic procedure (henceforth called the NOROBAIFEM-2D algorithm, without inverting the decomposition factor $T_{r}, 7$.

The challenge of computing parallel approximate inverses is to overcome its data dependencies, which create a critical path and an order of computations, hence any parallel approximate inverse matrix algorithm must abide by those dependencies in order to avoid any data loss.

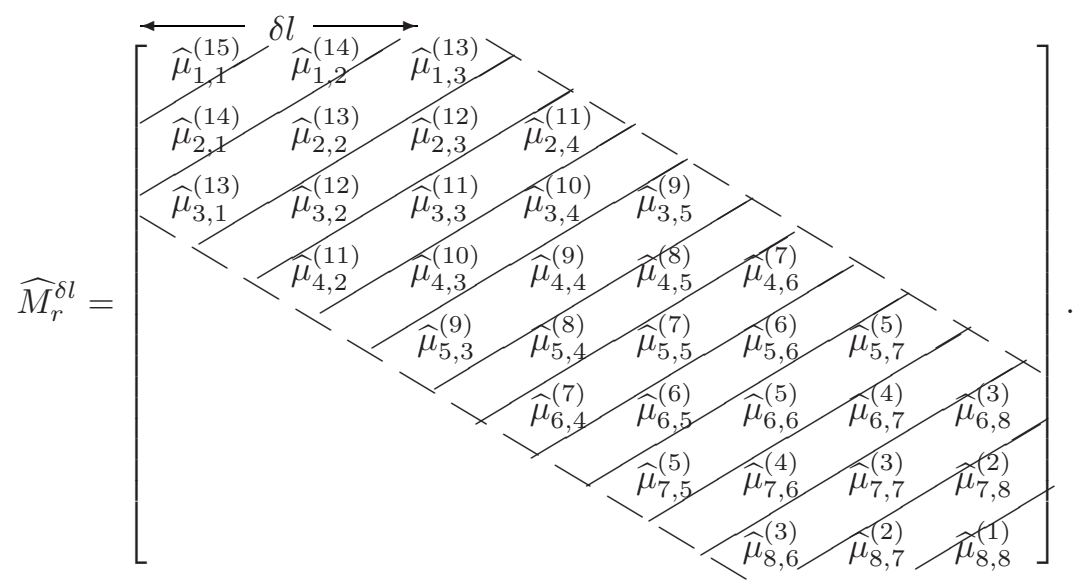

For the parallelization of the NOROBAIFEM-2D algorithm, an antidiagonal motion (wave-like pattern), starting from the element $\widehat{\mu}_{8,8}$ down to $\widehat{\mu}_{1,1}$, has been used, because of the dependency of the elements of the inverse during its construction. More specifically, any element within the banded approximate inverse requires its corresponding right or lower element to be computed first. This sequence of computations, without any loss of generality and for simplicity reasons, is shown for the normalized banded approximate inverse in equation (5) (with $n=8$ and $\delta l=3$ ). The values of the parentheses at the superscript of each element (e.g. $\left.\widehat{\mu}_{i, j}^{(k)}\right)$, indicate that the element $\widehat{\mu}_{i, j}$ was computed at the $(k)$-th sequential step of the algorithm ( $k$-th antidiagonal), while the elements with the same superscript (i.e. $(k)$ ) were computed concurrently. It should be noted that 
due to the data dependencies, for $\delta l=1,2$ the parallel algorithm will execute sequentially.

Let us consider that the command forall denotes the parallel for instruction (forks/joins threads), for executing parallel loops. Then, the algorithm for the implementation of the Parallel ANti Diagonal NOROBAIFEM-2D algorithm (henceforth called the PAND-NOROBAIFEM-2D algorithm), on symmetric multiprocessor systems, can be described as follows:

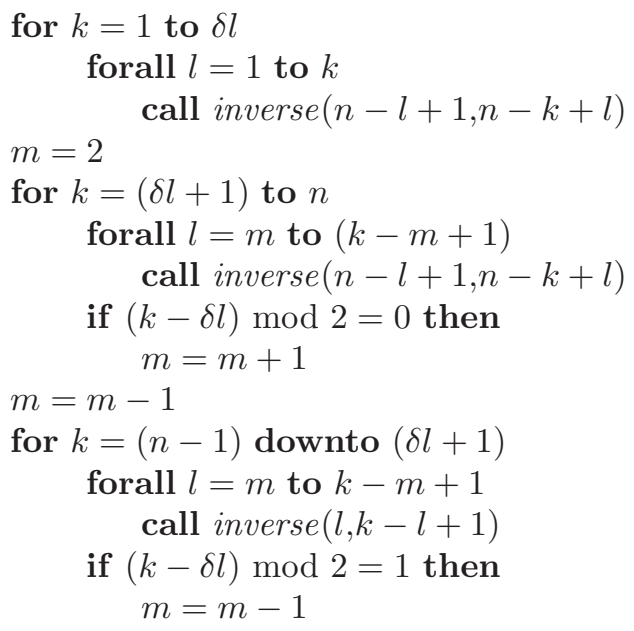

for $k=\delta l$ downto 1

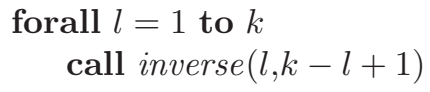

where the function inverse $(i, j)$ computes the element $\mu_{i, j}$ of the normalized optimized approximate inverse, and can be described as follows, [7]:

function(inverse)

Let $r \ell=r+\ell, m \ell=m+\ell, m r=m-r, n m r=n-m r, n m \ell=n-m \ell$.

if $\quad i>=j$ then

if $j>n m r$ then

if $i=j$ then

if $i=n$ then

$$
\widehat{\mu}_{1,1}=1
$$

else

else

$$
\widehat{\mu}_{n-i+1,1}=1-g_{j} \cdot \widehat{\mu}_{n-j, \delta l+1}
$$

else

$$
\widehat{\mu}_{n-i+1, i-j+1}=-g_{j} \cdot \widehat{\mu}_{n-i+1, i-j}
$$

if $j \geq r \ell$ and $j \leq n m r$ then

if $i=j$ then

$$
\begin{gathered}
\widehat{\mu}_{n-i+1,1}=1-g_{j} \cdot \widehat{\mu}_{n-j, \delta l+1}-\sum_{k=0}^{n m r-j} h_{r \ell-1-k, j+1-r+k} \cdot \widehat{\mu}_{x, y} \\
\operatorname{call} \mathbf{m w}(n, \delta l, i, m r+j+k, x, y)
\end{gathered}
$$


else

$$
\begin{aligned}
& \widehat{\mu}_{n-i+1, i-j+1}=-g_{j} \cdot \widehat{\mu}_{n-i+1, i-j}-\sum_{k=0}^{n m r-j} h_{r \ell-1-k, j+1-r+k} \cdot \widehat{\mu}_{x, y} \\
& \text { call } \mathbf{m w}(n, \delta l, i, m r+j+k, x, y) \\
& \text { else } \\
& \text { if } j>n m \ell+1 \text { and } j \leq r \ell-1 \text { then } \\
& \text { if } i=j \text { then } \\
& \widehat{\mu}_{n-i+1,1}=1-g_{j} \cdot \widehat{\mu}_{n-j, \delta l+1}-\sum_{\substack{k=j+1-r \\
k>0}}^{\ell} h_{j, k} \cdot \widehat{\mu}_{x_{1}, y_{1}} \\
& -\sum_{\lambda=0}^{n m \ell} h_{j-1-\lambda, \ell+1+\lambda} \cdot \widehat{\mu}_{x_{2}, y_{2}} \\
& \text { call } \mathbf{m w}\left(n, \delta l, i, m+k-1, x_{1}, y_{1}\right) \quad \text { call } \mathbf{m w}\left(n, \delta l, i, m \ell+\lambda, x_{2}, y_{2}\right) \\
& \text { else } \\
& \widehat{\mu}_{n-i+1, i-j+1}=-g_{j} \cdot \widehat{\mu}_{n-i+1, i-j}-\sum_{\substack{k=j+1-r \\
k>0}}^{\ell} h_{j, k} \cdot \widehat{\mu}_{x_{1}, y_{1}} \\
& -\sum_{\lambda=0}^{n m \ell} h_{j-1-\lambda, \ell+1+\lambda} \cdot \widehat{\mu}_{x_{2}, y_{2}} \\
& \widehat{\mu}_{n, 1}=1-g_{1} \cdot \widehat{\mu}_{n-1, \delta l+1}-\sum_{k=1}^{\ell} h_{1, k} \cdot \widehat{\mu}_{x, y} \\
& \text { call } \mathbf{m w}(n, \delta l, 1, m+k-1, x, y)
\end{aligned}
$$

$$
\widehat{\mu}_{n-i+1,1}=1-g_{j} \cdot \widehat{\mu}_{n-j, \delta l+1}-\sum_{\substack{k=j+1-r \\ k>0}}^{\ell} h_{j, k} \cdot \widehat{\mu}_{x_{1}, y_{1}}-\sum_{\lambda=1}^{j-1} h_{j-\lambda, \ell+\lambda} \cdot \widehat{\mu}_{x_{2}, y_{2}}
$$

call $\mathbf{m w}\left(n, \delta l, i, m+k-\ell, x_{1}, y_{1}\right) \quad$ call $\mathbf{m w}\left(n, \delta l, i, m \ell+\lambda-1, x_{2}, y_{2}\right)$

else

$$
\widehat{\mu}_{n-i+1,1}=-g_{j} \cdot \widehat{\mu}_{n-j, \delta l+1}-\sum_{\substack{k=j+1-r \\ k>0}}^{\ell} h_{j, k} \cdot \widehat{\mu}_{x_{1}, y_{1}}-\sum_{\lambda=1}^{j-1} h_{j-\lambda, \ell+\lambda} \cdot \widehat{\mu}_{x_{2}, y_{2}}
$$

call $\mathbf{m w}\left(n, \delta i, i, m+k-\ell, x_{1}, y_{1}\right) \quad$ call $\mathbf{m w}\left(n, \delta l, i, m \ell+\lambda-1, x_{2}, y_{2}\right)$

if $i<>j$ then

$$
\widehat{\mu}_{n-i+1, \delta l+i-j}=\widehat{\mu}_{n-i+1, i-j+1}
$$

The procedure $\operatorname{mw}(n, \delta l, s, q, x, y),[5]$, reduces the memory requirements of the approximate inverse to only $n \times(2 \delta l-1)$-vector spaces. The computational process is logically divided into $2 n-1$ sequential steps representing the $2 n-$ 1 antidiagonals, while synchronization between processes is needed after the 
computation of each antidiagonal, to ensure that the elements of the matrix are correctly computed.

\section{Parallel Normalized Preconditioned Conjugate Gradient method}

In this section we present a class of parallel Normalized Explicit Preconditioned Conjugate Gradient (NEPCG) method, based on the derived parallel optimized approximate inverse, designed for symmetric multiprocessor systems.The NEPCG method for solving linear systems has been presented in [7]. The computational complexity of the NEPCG method is $O[(2 \delta l+2 \ell+11) n$ mults $+3 n$ adds] $\nu$ operations, where $\nu$ is the number of iterations required for the convergence to a certain level of accuracy, 7.

The Parallel Normalized Explicit Preconditioned Conjugate Gradient (PN EPCG) algorithm for solving linear systems can then be described as follows:

$$
\begin{aligned}
& \text { forall } j=1 \text { to } n \\
& \qquad\left(r_{0}\right)_{j}=s_{j}-A\left(u_{0}\right)_{j}
\end{aligned}
$$

if $\delta l=1$ then

$$
\begin{aligned}
& \text { forall } j=1 \text { to } n \\
& \qquad\left(r_{0}^{*}\right)_{j}=\left(r_{0}\right)_{j} /\left(d^{2}\right)_{j}
\end{aligned}
$$

else

$$
\begin{aligned}
& \text { forall } j=1 \text { to } n \\
& \qquad \begin{array}{r}
\left(r_{0}^{*}\right)_{j}=\left(\sum_{k=\max (1, j-\delta l+1)}^{j} \widehat{\mu}_{n+1-i, i+1-k}\left(r_{0}\right)_{k} / d_{k}\right. \\
\left.+\sum_{k=j+1}^{\min (n, j+\delta l-1)} \widehat{\mu}_{n+1-k, \delta l+k-j}\left(r_{0}\right)_{k} / d_{k}\right) /(d)_{j}
\end{array}
\end{aligned}
$$

forall $j=1$ to $n$

$$
\left(\sigma_{0}\right)_{j}=\left(r_{0}^{*}\right)_{j}
$$

forall $j=1$ to $n$ (reduction $\left.+p_{0}\right)$

$$
p_{0}=\left(r_{0}\right)_{j} *\left(r_{0}^{*}\right)_{j}
$$

Then, for $i=0,1, \ldots$, (until convergence) compute in parallel the vectors $u_{i+1}$, $r_{i+1}, \sigma_{i+1}$ and the scalar quantities $\alpha_{i}, \beta_{i+1}$ as follows:

forall $j=1$ to $n$

$$
\left(q_{i}\right)_{j}=A\left(\sigma_{i}\right)_{j}
$$

forall $j=1$ to $n$ (reduction $+t_{i}$ )

$$
\begin{aligned}
& \quad t_{i}=\left(\sigma_{i}\right)_{j} *\left(q_{i}\right)_{j} \\
& \alpha_{i}=p_{i} / t_{i}
\end{aligned}
$$

forall $j=1$ to $n$

$$
\begin{aligned}
& \left(u_{i+1}\right)_{j}=\left(u_{i}\right)_{j}+\alpha_{i}\left(\sigma_{i}\right)_{j} \\
& \left(r_{i+1}\right)_{j}=\left(r_{i}\right)_{j}-\alpha_{i}\left(q_{i}\right)_{j}
\end{aligned}
$$

if $\delta l=1$ then

$$
\begin{aligned}
& \text { forall } j=1 \text { to } n \\
& \qquad\left(r_{i+1}^{*}\right)_{j}=\left(r_{i+1}\right)_{j} /\left(d^{2}\right)_{j}
\end{aligned}
$$


else

$$
\text { forall } j=1 \text { to } n
$$

$$
\begin{aligned}
&\left(r_{i+1}^{*}\right)_{j}=\left(\sum_{k=\max (1, j-\delta l+1)}^{j} \widehat{\mu}_{n+1-i, i+1-k}\left(r_{i+1}\right)_{k} / d_{k}\right. \\
&\left.+\sum_{k=j+1}^{\min (n, j+\delta l-1)} \widehat{\mu}_{n+1-k, \delta l+k-j}\left(r_{i+1}\right)_{k} / d_{k}\right) /(d)_{j}
\end{aligned}
$$

forall $j=1$ to $n$ (reduction $\left.+p_{i+1}\right)$

$$
\begin{aligned}
& \qquad p_{i+1}=\left(r_{i+1}\right)_{j} *\left(r_{i+1}^{*}\right)_{j} \\
& \beta_{i+1}=p_{i+1} / p_{i} \\
& \text { forall } j=1 \text { to } n \\
& \qquad\left(\sigma_{i+1}\right)_{j}=\left(r_{i+1}^{*}\right)_{j}+\beta_{i+1}\left(\sigma_{i}\right)_{j}
\end{aligned}
$$

It should be noted that the parallelization of the coefficient matrix $A \times$ vector operation has been implemented by taking advantage of the sparsity of the coefficient matrix $A$.

\section{Numerical Results}

In this section we examine the applicability and effectiveness of the proposed parallel schemes for solving sparse finite element linear systems.

Let us now consider a 2D-boundary value problem:

$$
u_{x x}+u_{y y}+u=F, \quad(x, y) \in R, \quad \text { with } \quad u(x, y)=0, \quad(x, y) \in \partial R,
$$

where $R$ is the unit square and $\partial R$ denotes the boundary of $R$.

The domain is covered by a non-overlapping triangular network resulting in a hexagonal mesh. The right hand side vector of the system (1) was computed as the product of the matrix $A$ by the solution vector, with its components equal to unity. The "fill-in" parameter was set to $r=2$ and the width parameter was set to $\ell=3$. The iterative process was terminated when $\left\|r_{i}\right\|_{\infty}<10^{-5}$. It should be noted that further details about the convergence behavior and the impact of the "retention" parameter on the solution can be found in [6].

The numerical results presented in this section were obtained on an SMP machine consisting of $162.2 \mathrm{GHz}$ Dual Core AMD Opteron processors, with 32 GB RAM running Debian GNU/Linux (National University Ireland Galway). For the parallel implementation of the algorithms presented, the Intel C Compiler v9.0 with OpenMP directives has been utilized with no optimization enabled at the compilation level. It should be noted that due to administrative policies, we were not able to explore the full processor resources (i.e. more than 8 threads).

In our implementation, the parallel for pragma has been used in order to generate code that forks/joins threads, in all cases. Additionally, static scheduling has been used (schedule(static)), whereas the use of dynamic scheduling has not produced improved results.

The speedups and efficiencies of the PAND-NOROBAIFEM-2D algorithm for several values of the "retention" parameter $\delta l$ with $n=10000$ and $m=101$, 
Table 1. Speedups for the PAND-NOROBAIFEM-2D algorithm for several values of $\delta l$

\begin{tabular}{cccc}
\hline \multicolumn{4}{c}{ Speedups for the PAND-NOROBAIFEM-2D algorithm } \\
\hline \multicolumn{4}{c}{ Retention parameter 2 } \\
\hline$\delta l=m$ & 1.8966 & 3.8458 & 6.8653 \\
\hline$\delta l=2 m$ & 1.9600 & 3.8505 & 7.4011 \\
\hline$\delta l=4 m$ & 1.9741 & 3.9260 & 7.5768 \\
\hline$\delta l=6 m$ & 1.9986 & 3.9501 & 7.8033 \\
\hline
\end{tabular}

Table 2. Efficiencies for the PAND-NOROBAIFEM-2D algorithm for several values of $\delta l$

\begin{tabular}{|c|c|c|c|}
\hline \multicolumn{4}{|c|}{ Efficiencies for the PAND-NOROBAIFEM-2D algorithm } \\
\hline Retention parameter 2 & processors 4 & processors 8 & processors \\
\hline$\delta l=m$ & 0.9483 & 0.9615 & 0.8582 \\
\hline$\delta l=2 m$ & 0.9800 & 0.9626 & 0.9251 \\
\hline$\delta l=4 m$ & 0.9870 & 0.9815 & 0.9471 \\
\hline$\delta l=6 m$ & 0.9993 & 0.9875 & 0.9754 \\
\hline
\end{tabular}

are given in Table 1 and 2. In Fig. 1 the parallel speedups for several values of the "retention" parameter $\delta l$ are presented for the PAND-NOROBAIFEM2D method, for $n=10000$ and $m=101$.

The speedups and efficiencies of the PNEPCG algorithm for several values of the "retention" parameter $\delta l$ with $n=10000$ and $m=101$, are given in Table 3 and 4 . In Fig. 2 the parallel speedups for several values of the "retention" parameter $\delta l$ are presented for the PNEPCG method, for $n=10000$ and $m=101$.

Table 3. Speedups for the PNEPCG algorithm for several values of $\delta l$

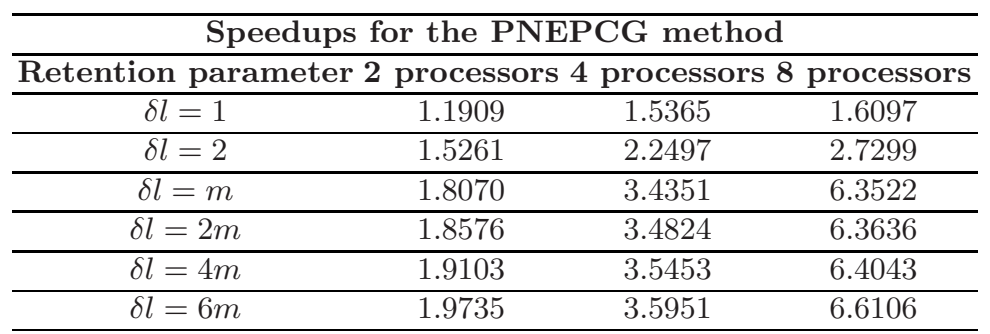

It should be mentioned that for large values of the "retention" parameter, i.e. multiples of the semi-bandwidth $m$, the speedups and the efficiency tend to the upper theoretical bound, for both the parallel construction of the approximate inverse and the parallel normalized preconditioned conjugate gradient method, since the coarse granularity amortizes the parallelization overheads. For small 


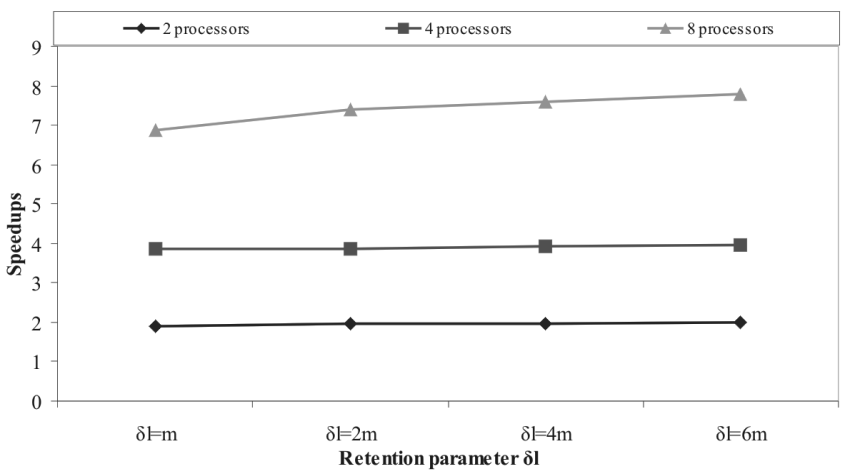

Fig. 1. Speedups versus the "retention"parameter $\delta l$ for the PANDNOROBAIFEM-2D algorithm

Table 4. Efficiencies for the PNEPCG algorithm for several values of $\delta l$

\begin{tabular}{|c|c|c|c|}
\hline \multicolumn{4}{|c|}{ Efficiencies for the PNEPCG algorithm } \\
\hline Retention parameter 2 & process & rocess & processors \\
\hline$\delta l=1$ & 0.5955 & 0.3841 & 0.2012 \\
\hline$\delta l=2$ & 0.7631 & 0.5624 & 0.3412 \\
\hline$\delta l=m$ & 0.9035 & 0.8588 & 0.7940 \\
\hline$\delta l=2 m$ & 0.9288 & 0.8706 & 0.7954 \\
\hline$\delta l=4 m$ & 0.9551 & 0.8863 & 0.8005 \\
\hline$\delta l=6 m$ & 0.9867 & 0.8988 & 0.8263 \\
\hline
\end{tabular}

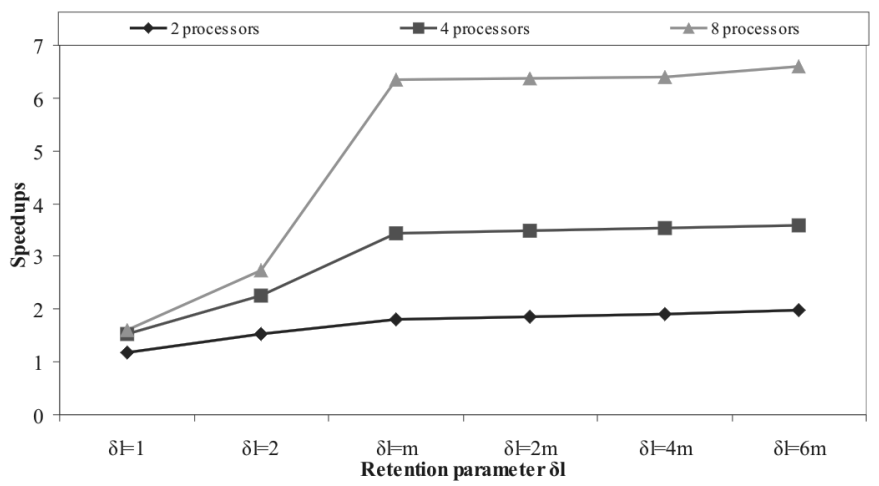

Fig. 2. Speedups versus the "retention" parameter $\delta l$ for the PNEPCG algorithm

values of the "retention" parameter, i.e. $\delta l=1,2$, the fine granularity is responsible for the low parallel performance, since the parallel operations reduces to simple ones, like inner products, and the utilization of the hardware platform is decreasing. 


\section{Conclusion}

The design of parallel explicit approximate inverses results in efficient parallel preconditioned conjugate gradient method for solving finite element linear systems on multiprocessor systems.

Finally, further parallel algorithmic techniques will be investigated in order to improve the parallel performance of the normalized explicit approximate inverse preconditioning on symmetric multiprocessor systems, particularly by increasing the computational work output per processor and eliminating process synchronization and any associated latencies.

Acknowledgments. The author would like to thank indeed Dr. John Morrison of the Department of Computer Science, University College of Cork for the provision of computational facilities and support through the WebCom-G project funded by Science Foundation Ireland.

\section{References}

1. Akl, S.G.: Parallel Computation: Models and Methods. Prentice-Hall, Englewood Cliffs (1997)

2. Dongarra, J.J., Duff, I., Sorensen, D., van der Vorst, H.A.: Numerical Linear Algebra for High-Performance Computers. SIAM, Philadelphia (1998)

3. Duff, I.: The impact of high performance computing in the solution of linear systems: trends and problems. J. Comp. Applied Math. 123, 515-530 (2000)

4. Gravvanis, G.A.: Explicit Approximate Inverse Preconditioning Techniques. Archives of Computational Methods in Engineering 9(4), 371-402 (2002)

5. Gravvanis, G.A.: Parallel matrix techniques. In: Papailiou, K., Tsahalis, D., Periaux, J., Hirsch, C., Pandolfi, M. (eds.) Computational Fluid Dynamics I, pp. 472-477. Wiley, Chichester (1998)

6. Gravvanis, G.A., Giannoutakis, K.M.: Normalized Explicit Finite Element Approximate Inverses. I. J. Differential Equations and Applications 6(3), 253-267 (2003)

7. Gravvanis, G.A., Giannoutakis, K.M.: Normalized finite element approximate inverse preconditioning for solving non-linear boundary value problems. In: Bathe, K.J. (ed.) Computational Fluid and Solid Mechanics 2003. Proceedings of the Second MIT Conference on Computational Fluid and Solid Mechanics, vol. 2, pp. 1958-1962. Elsevier, Amsterdam (2003)

8. Grote, M.J., Huckle, T.: Parallel preconditioning with sparse approximate inverses. SIAM J. Sci. Comput. 18, 838-853 (1977)

9. Huckle, T.: Approximate sparsity patterns for the inverse of a matrix and preconditioning. Applied Numerical Mathematics 30, 291-303 (1999)

10. Saad, Y., van der Vorst, H.A.: Iterative solution of linear systems in the 20th century. J. Comp. Applied Math. 123, 1-33 (2000) 